\title{
O uso do Ecomapa na saúde como ferramenta pedagógica: um relato de experiência
}

\author{
The use of Ecomapa in health as a pedagogical tool: an experience report
}

El uso de Ecomapa en salud como herramienta pedagógica: un informe de experiencia

\begin{abstract}
Domitília Bonfim de Macêdo Mihaliuc ${ }^{1 *}$, Bárbara Louise Moreira Silva², Lúcia Helena Bueno da Fonseca $^{1}$, Walterlânia Silva Santos².
\end{abstract}

\section{RESUMO}

Objetivo: Relatar a experiência docente na utilização do ecomapa, outrora empregado no âmbito da saúde, como ferramenta pedagógica de autoavaliação discente. Relato de experiência: Trata-se de um relato de experiência, liderado por uma docente atuante de uma faculdade pública do Distrito Federal sobre a autoutilização do ecomapa em sala virtual com 47 discentes, de ambos os sexos, os quais cursavam o último ano de graduação em Enfermagem. Assim, a docente orientou a realização de uma atividade, para que os estudantes elaborassem dois ecomapas individuais. O primeiro retrataria suas relações antes do ensino remoto emergencial, já o segundo, o momento presente. Após isso, a docente refletiu sobre a ferramenta para compreender os vínculos dos discentes, e nortear suas práticas pedagógicas; sendo que a maioria tem fragilidades ou rompimento de vínculos. Considerações finais: Há a possibilidade de transferência de aplicação do ecomapa do setor da saúde para o acadêmico, como instrumento alternativo e inovador à reorganização docente no processo de desenvolvimento de conexões interpessoais no modelo de ensino virtual.

Palavras-chave: Educação em enfermagem, Docentes, Educação à distância, Educação superior, Enfermagem.

\begin{abstract}
Objective: To report the teaching experience in using the eco-map, formerly used in the health field, as a pedagogical tool for student self-assessment. Experience report: This is an experience report, led by an professor at a public university in the Brasília (capital of Brazil), about the self-use of the eco-map in a virtual classroom with 47 students, of both sexes, who were in the last year of graduation in Nursing. Thus, the professor guided the performance of an activity, so that students could prepare two individual ecomaps. The first had portrayed their relationships before the remote teaching, the second, the present moment. After that, the professor reflected on the tool to understand the bonds of students and guide their pedagogical practices; most of them have weaknesses or broken ties. Final considerations: There is the possibility of transferring the application of the eco-map from the health sector to the academic sector, as an alternative and innovative instrument to the reorganization of professors in the process of developing interpersonal connections in the virtual teaching model.
\end{abstract}

Key words: Education nursing, Faculty, Education distance, Education higher, Nursing.

${ }^{1}$ Secretaria de Saúde do Distrito Federal, Brasília - DF

*E-mail: domi.bonfim@gmail.com

2 Universidade de Brasília, Brasília - DF.

SUBMETIDO EM: 8/2021 


\section{RESUMEN}

Objetivo: Informar la experiencia docente en el uso del ecomapa, utilizado en el campo de la salud, como herramienta pedagógica para la autoevaluación del alumno. Relato de experiencia: Se trata de un informe de experiencia, dirigido por un profesor de una universidad pública del Brasília (capital de Brasil), sobre el uso propio del ecomapa en un aula virtual con 47 estudiantes, de ambos sexos, que estaban en el último año de graduación en Enfermería. Así, el profesor orientó la realización de una actividad, para que los alumnos pudieran elaborar dos ecomapas individuales. El primero había retratado sus relaciones antes de la enseñanza a distancia, el segundo, el momento presente. Posteriormente, el docente reflexionó sobre la herramienta para comprender los vínculos de los estudiantes y orientar sus prácticas pedagógicas; la mayoría tiene debilidades o vínculos rotos. Consideraciones finales: Existe la posibilidad de trasladar la aplicación del ecomapa del sector salud al sector académico, como un instrumento alternativo e innovador a la reorganización de los docentes en el proceso de desarrollo de conexiones interpersonales en el modelo de enseñanza virtual.

Palabras clave: Educación en enfermería, Docentes, Educación a distancia, Educación superior, Enfermería.

\section{INTRODUÇÃO}

No contexto hodierno, caracterizado pela pandemia causada pelo SARS-CoV-2, o ensino acadêmico passa por mudanças tempestivas, dentre as quais, a substituição das aulas presenciais por aulas em meios digitais, orientadas inicialmente pela Portaria $n^{\circ} 343 / 2020$ do Ministério da Educação (MEC), e com prazo estendido pela Portaria MEC ํ5 544/20 para o período que durar a pandemia (2020) (MINISTÉRIO DA EDUCAÇÃO, 2020).

A essa conjuntura, destaca-se as Diretrizes Curriculares Nacionais (DCN) dos cursos de graduação da área da saúde abordam princípios e regulamentos resultantes de uma construção coletiva e democrática. Outrossim, as DCN da Enfermagem definem princípios, fundamentos e condições do processo de formação acadêmica, assim como, estabelecem competências e habilidades gerais que devem ser desenvolvidas pelo futuro enfermeiro, sendo elas: atenção à saúde, tomada de decisões, administração e gerenciamento, comunicação, liderança e educação permanente (MINISTÉRIO DA SAÚDE, 2018).

O aprimoramento das competências supracitadas não ocorre somente a partir de práticas pedagógicas, que incluem atividades didáticas. $O$ docente media o processo, principalmente, no que tange à formação dinâmica, humanística, crítica e reflexiva do discente de enfermagem (NETTO L e SILVA KL, 2018). Nesse cenário, indica-se a implementação de tecnologias educacionais incorporadas ao âmbito acadêmico como estratégia de ensino que sejam capazes de auxiliar o discente no processo de aprendizagem ativa, além de, viabilizar estreitamento de relações entre o docente e o discente, mesmo na condição estabelecida de ensino virtual (COSTA BCP, et al., 2021).

Essa abordagem torna-se relevante, tendo em vista que o ensino por meio digital envolve, não apenas, aspectos espaciais e temporais, mas também questões psicossociais, quando se avaliam relações afetivas e interativas inclusas nessa modalidade de ensino (ANTUNES JT e BATISTA PVC, 2016). Em síntese, evidencia-se a pertinência da atuação do docente como um mediador de estratégias de planejamento e de execução, as quais objetivem o estabelecimento de tais vínculos sociais, análogo à conjuntura do Ambiente Virtual Acadêmico (AVA). Em conformidade ao exposto, nota-se o uso das Tecnologias de Informação e Comunicação (TICs) como forma de possibilitar a minimização de interferências negativas nas relações interpessoais entre os acadêmicos, de modo a auxiliar no processo de formação (FETTERMANN FA, et al., 2019).

Assim, a modalidade de autoaplicação de instrumentos como modo de concretizar a apropriação do aprendizado pode ser utilizada, principalmente, com orientações de manutenção de distanciamento social e atividades remotas. Dessa forma, apesar do ecomapa ser recomendado para a avaliação das relações e 
vínculos do usuário vulnerável na área da saúde, e refletindo que no ensino por meio digital, há um movimento crescente de criar estratégias para resgatar as relações docente-discente, lançou-se a proposta de autoaplicação do ecomapa para estudantes de enfermagem (MINISTÉRIO DA SAÚDE, 2013).

Cabe ressaltar que o ecomapa tem a finalidade de promover compreensão e avaliação das relações individuais com a esfera social, por meio da análise esquemática das estruturas internas e externas de determinada família, utente ou ofício. Para tal fim, representações gráficas equivalem as ligações, interações e relações estabelecidas juntamente ao meio social em análise (COSTA PHA, et al., 2016).

Essa atividade de transpor teoria para a prática nos cursos da área da saúde acontecerá imprescindivelmente, uma vez que o ensino digital se concentrou em atividades teóricas (MINISTÉRIO DA EDUCAÇÃO, 2020).

$\mathrm{Na}$ experiência descrita a seguir, a docente teve o intuito de compreender aspectos relacionados aos vínculos dos discentes, de maneira a potencializar características individuais e grupais para 0 desenvolvimento de competências. Desse modo, o objetivo deste artigo foi relatar a experiência docente na utilização do ecomapa, outrora empregado no âmbito da saúde, como ferramenta pedagógica de autoavaliação discente, visto que o momento pandêmico exige novas formas de construir processos de ensino-aprendizagem.

\section{RELATO DE EXPERIÊNCIA}

A experiência aconteceu no mês de abril de 2021 em sala virtual com 47 discentes que cursavam último ano de graduação em Enfermagem, liderada por docente com 12 anos de experiência em ensino presencial, de faculdade pública do Distrito Federal. Durante oficina sobre saúde do idoso, a docente apresentou ferramentas de avaliação do idoso vulnerável, dentre estas, o ecomapa. Ao final da atividade, propôs que cada discente elaborasse dois ecomapas referentes a si próprios, um correspondente ao período atual e outro referente a antes do contexto pandêmico, também como forma de dar mais significado ao aprendizado.

Apesar da proposta não se configurar como avaliação somativa na nota final, os discentes enviaram os arquivos por meio de ambiente virtual compartilhado, 48 horas após a oficina, totalizando 94 arquivos recebidos.

Após sete dias da proposta inicial, a docente reuniu em ambiente virtual novamente com os discentes, para compartilhar sobre a construção dos ecomapas, e questionou como essa atividade significou para compreensão dessa ferramenta. A partir disso, a docente defrontou-se com uma estratégia pedagógica para que os discentes se autoavaliassem quanto ao entendimento de ferramenta para abordagem familiar em condição vulnerável, ao tempo que também visualizou a autorreflexão como pessoas que perpassavam por período de diminuição de intensidade e de quantidade das interações sociais.

A docente percebeu na entonação de voz, gestos faciais, assim como na mensagem falada, reações de surpresa, tristeza, que alguns expressaram com lágrimas, como também a identificação entre os presentes, já que a maioria se encontrava em situação similar. Vale ressaltar que pactuaram que todos permanecessem com as câmeras acionadas durante o encontro virtual, e dessa forma, foi possível observar esse movimento grupal.

Ao observar cada desenho, um anterior à pandemia e o outro atual, percebeu-se perdas de relações sociais e afetivas dos discentes, inclusive com a instituição de ensino e seus atores (docentes, coordenador, monitores, colegas). Portanto, a docente refletiu sobre a necessidade de planejar atividades para atender a essas demandas, que inclui discentes que podem não ter se adaptado ao ensino digital, ou que não identificaram relação satisfatória com a instituição, mesmo com atividades remotas diárias.

Partindo do pressuposto de que identificar fragilidades e potencialidades de cada indivíduo de forma digital é um problema para o professor no contexto da pandemia, a avaliação dos ecomapas dos discentes superou a expectativa inicial, que era proporcionar uma aprendizagem significativa, e trouxe informações preciosas para que o docente pudesse compreender o universo estudantil nessas circunstâncias. 
Figura 1 - Ecomapas simulados para estudante no período pré e pós-pandemia covid-19.

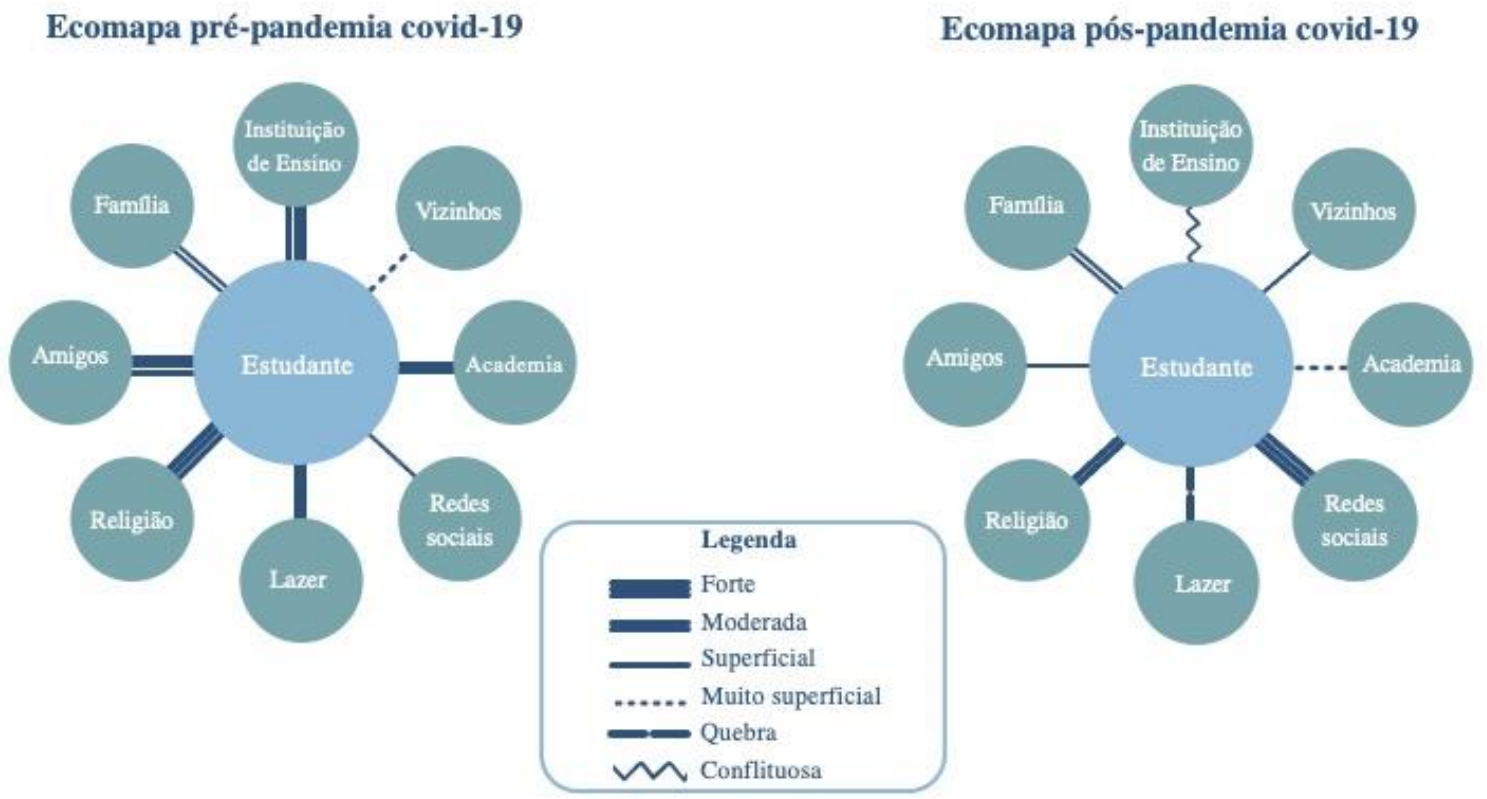

Nota: Imagem produzida na plataforma Canva®. Fonte: Mihaliuc DBM, et al., 2021.

\section{DISCUSSÃO}

A experiência permitiu utilizar com êxito um instrumento de avaliação da área da saúde na educação, com o mesmo objetivo, mas para qualificar o docente no planejamento e/ou replanejamento de suas práticas pedagógicas. Foi possível também estabelecer vínculo com estudantes, mesmo de forma remota, quando o professor, por meio do ecomapa, compreende seus contextos de vida.

Para o docente, o ecomapa transformou-se em ferramenta pedagógica de qualificação, de maneira que seus resultados impactaram no planejamento das atividades futuras. Ao mesmo tempo percebeu que 0 distanciamento social influenciou na vida de cada discente, surgiu então um alerta a comunidade docente para novos procedimentos e técnicas no sentido de corroborar no processo de ensino-aprendizagem de estudantes da saúde.

Sabe-se que o Estágio Curricular Supervisionado (ECS) promove ao estudante o desenvolvimento de autonomia, rigor técnico-científico, gestão e gerenciamento de recursos, proatividade, liderança, dentre outras potencialidades. Assim, compreende-se que práticas baseadas em evidências oportunizam o aprimoramento de competências, de forma a assegurar a integralidade à atenção à saúde de indivíduos, grupos sociais e coletividades. No entanto, nota-se que o ensino remoto, além de impossibilitar a realização do ECS, carrega particularidades ambientais, atitudinais e sociais. Desse modo, torna-se essencial a implementação de ferramentas inovadoras que sejam acessíveis aos discentes e aos docentes (ARAÚJO AAC, et al., 2020).

Diante do exposto, percebe-se que a conjuntura atual, possivelmente, desencadeará prejuízos quanto ao progresso intelectual e profissional autônomo e continuado do estudante vigente, de modo a interferir na garantia de uma atenção integralizada. Tal fato se deve a essa nova realidade social, marcada por mudanças educacionais, as quais têm desencadeado impactos individuais e coletivos negativos. Posto isto, entende-se que as atuais modalidades de ensino demandam de processos adaptativos, de maneira a visar a continuidade do sistema de ensino-aprendizagem a partir de manutenções efetivas e satisfatórias da educação ofertada (RAMOS TH, et al., 2020).

Sendo assim, entende-se que docência mediada por tecnologia concedeu maior flexibilidade nos métodos formativos, de modo a auxiliar no processo de atendimento das novas demandas sociais. Outrossim, salientase que essa era tecnológica viabilizou a ocorrência de inovações procedimentais de aprendizagem, as quais tendem a contribuir no alcance de uma abordagem acadêmica colaborativa (CARNEIRO LA, et al., 2020). 
Dessa forma, nota-se que a incorporação de tecnologias digitais, no ambiente educacional, tornou-se crucial no suporte, gerenciamento e armazenamento do sistema de ensino-aprendizagem colaborativamente desenvolvido (SALES AB e BOSCARIOLI C, 2020). Portanto, além do ecomapa referido no decorrer deste artigo, cabe citar outros viáveis instrumentos tecnológicos, tem-se como exemplo desses o Jamboard $\Theta$, o Padlet $\AA^{\circledR}$, o Google Classroom $\AA$, Canva for Education ${ }^{\circledR}$, Mentimeter ${ }^{\circledR}$, dentre outros. Conforme Sales AB e Boscarioli C (2020), observa-se que a aplicação de ferramentas colaborativas e engajadoras na docência potencializa a inserção do modelo de metodologia ativa no cenário acadêmico.

Diante do contexto de aulas digitais, o diálogo entre docente e discentes restringe-se quase que somente à assuntos pedagógicos. $O$ estudante, com todo seu dinamismo e contexto social, tem mais dificuldade de estabelecer vínculo com o professor (RAMÔA HN, et al., 2020). Assim, o desenvolvimento de habilidades e de atitudes ficou mais desafiador para o docente de enfermagem por meio de aulas digitais. Ademais, inferese que nem todos os estudantes se adaptaram ao ensino digital e podem estar em sofrimento ou desmotivados. Posto isto, observa-se que o ensino remoto traz uma problemática a partir da utilização das ferramentas de plataformas digitais, tendo em vista o acarretamento de interferências nas esferas cognitivas e afetivas dos discentes e docentes (CIPRIANO JA e ALMEIDA LCCS, 2020).

Nesse ínterim, Arruda EP (2020) destaca o conceito de Ensino Remoto Emergencial (ERE) como sendo uma mudança temporária no modo de oferta dos conteúdos curriculares, assim, esses podem ser disponibilizados por meio de plataformas digitais on-line, as quais permitam a participação simultânea dos indivíduos envolvidos, ou podem ser oferecidos a partir de materiais e atividades assíncronas. Neste âmbito, Santos GMRF (2021) complementa tal entendimento quando explana que a continuidade das práticas educativas se deu por meio do ERE alicerçado ao desempenho das TICs. Por fim, é crucial ressaltar que o ensino referenciado possibilita um maior alcance temporal e geográfico, sendo estes fatores oportunos à virtualização do processo educativo de maneira ágil, frente às novas condições estabelecidas.

Paralelo ao evidenciado, nota-se que, a partir das drásticas mudanças nas condições educacionais, surgiram novos desafios pedagógicos, de modo a demandar dos docentes ações e atitudes adaptativas (SILVA MD, et al., 2021). Este processo estimulou alguns docentes ao desenvolvimento de inovações educativas frente às demandas antes irrefletidas. Dessa forma, entende-se que o aprimoramento de competências científicas e pedagógicas por parte dos professores, tornou-se de suma importância ao desenvolvimento, adesão e aplicação de atualizadas estratégias acadêmicas (RIEGEL F, et al., 2021).

Em síntese, nota-se que o advento da pandemia de COVID-19 levantou a necessidade de uma abordagem docente inovadora, a qual possibilitasse a percepção das fragilidades correntes dos discentes. Tal feito tem a intencionalidade de criar vínculos entre professor e estudante, de modo que esta circunstância permita a compreensão contextual de cada discente, viabilizando, assim, reorganização de novas condutas pedagógicas. Nesse cenário, traz-se, ao setor acadêmico, a implementação do ecomapa, podendo ser ferramentas tecnológicas ajustada à esfera atual.

Ressalta-se que tecnologia compreende saberes, que podem ser agrupados em três categorias: tecnologia dura, retratada pelo material concreto, como equipamentos e maquinários; tecnologia leve-dura, representada pelos saberes estruturados que direcionam o trabalho, ou seja, as teorias, as normas, o conhecimento produzido em setores específicos; e tecnologia leve, que se destaca como processo de produção da comunicação, das relações, dos vínculos, sendo esses atributos das relações humanas (MERHY EE, et al., 2016; SABINO LMM, et al., 2016).

Assim, o ecomapa é tecnologia leve-dura, em que, quando inserida no ambiente acadêmico, possibilita tanto a autoavaliação e a autorreflexão discente, como a qualificação docente. Como também pode se comportar como tecnologia leve, por proporcionar estreitamento de vínculos professor-estudante, seguida da compreensão contextual, de modo a propiciar ao profissional o planejamento pertinente. Por conseguinte, nota-se que essa experiência oportunizou identificação de fragilidades individuais, despertando assim o desenvolvimento de novas técnicas educacionais que visam minimizar prováveis inconvenientes decorrentes do ensino virtual, tal como prejuízo no aprimoramento de competências e habilidades requeridas no campo de trabalho. 
A partir da observação dos símbolos gráficos dos ecomapas dos estudantes, que representam a intensidade da relação existente ou perdida com outras pessoas ou instituições, percebeu-se que, após um ano de isolamento social, 39 (83\%) estudantes tiveram repercussões negativas em suas relações sociais. Quanto à relação dos estudantes com a instituição de ensino superior, 29 (62\%) estudantes tiveram suas relações modificadas após ensino não presencial.

Sugere-se que outros estudos sejam realizados para que se valide e consolide o uso dessa ferramenta como estratégia pedagógica de reconhecer a rede social de discentes, com a possibilidade de favorecer a aproximação docentes-discentes, além de despertar para aspectos que têm potencial de provocar prejuízos no processo de ensino-aprendizagem.

Esta experiência promoveu reflexões e identificou a necessidade de reformular e de inovar as práticas de ensino remotas, ajudou a compreender o impacto do isolamento social na relação com a instituição de ensino superior. Desse modo, além de exercitar o uso dessa ferramenta, a docente pôde visualizar as diferenças contextuais dos discentes que realizarão os atendimentos presenciais aos usuários nos serviços de saúde após longo período de atividades remotas.

Consoante ao exposto, nota-se que o momento exige reorganização e treinamento docente, principalmente, da área da saúde, de maneira a manter uma interatividade contínua com o discente, desfrutar de tecnologias de ensino e, ainda assim, desenvolver competências profissionais.

Destarte, para manter a formação acadêmica em saúde voltada para o desenvolvimento de competências, e diante da interação docente-discente virtual, faz-se necessário uso de ferramentas alternativas capazes de promover aproximação entre os protagonistas do processo ensino-aprendizagem. Sendo que o ecomapa, utilizado na área da saúde como ferramenta de avaliação do usuário, pode se apresentar como instrumento inovador para que o docente compreenda a subjetividade do discente em plena crise sanitária, outrora percebida durante as aulas presenciais. Portanto, esta experiência positiva da utilização do ecomapa no contexto pedagógico pode despertar a comunidade científica sobre a necessidade emergencial de construir novas maneiras de promover engajamento, interatividade e vínculo no ambiente de sala de aula, virtual ou presencial.

\section{REFERÊNCIAS}

1. ANTUNES JT, BATISTA PVC. EAD e desafios de interação: um estudo de revisão. Revista Multitexto, 2016; 1(4): 32-36.

2. ARAÚJO AAC, et al. O ensino de graduação em enfermagem durante a pandemia da COVID-19. Revista Cuidarte, 2020; 12(1): 1-3.

3. ARRUDA EP. Educação remota emergencial: elementos para políticas públicas na educação brasileira em tempos de Covid-19. Revista de Educação a Distância, 2020; 1(7): 257-275.

4. BACICH L, MORAN J. Metodologias ativas para uma educação inovadora: uma abordagem teórico-prática. Porto Alegre: Penso, 2018; $430 \mathrm{p}$.

5. CARNEIRO LA, et al. Uma revisão sobre aprendizagem colaborativa mediada por tecnologias. Revista Desafios, 2020; 2(7): 52-62.

6. CIPRIANO JA, ALMEIDA LCCS. Educação em tempos de pandemia: análises e implicações na saúde mental do professor e aluno. 2020.

7. COSTA BCP, et al. Tecnologia em saúde e sua influência no ensino em enfermagem. Cuidado é Fundamental, 2021; 13: 288-294.

8. COSTA PHA, et al. O ecomapa como ferramenta na formação para o trabalho em rede no campo de álcool e outras drogas. Revista Pesquisas e Práticas Psicossociais, 2016; 3(11): 669-681.

9. FETTERMANN FA, et al. A utilização do Hangout como estratégia de ensino na formação de professores. Revista Eletrônica Acervo Saúde, 2019; 11(7): e609.

10. MERHY EE, et al. Avaliação compartilhada do cuidado em saúde: surpreendendo o instituído nas redes. 1 ed. Rio de Janeiro: Hexis, 2016; 448 p.

11. MINISTÉRIO DA SAÚDE. Conselho Nacional de Saúde. Resolução № 573, de 31 de janeiro de 2018. 2018. Diretrizes Curriculares Nacionais (DCN) para o curso de graduação Bacharelado em Enfermagem. Brasil, 2018. Disponível em: https://www.in.gov.br/materia/-/asset_publisher/Kujrw0TZC2Mb/content/id/48743098/do1-2018-11-06-resolucao-n573-de-31-de-janeiro-de-2018-48742847. Acessado em: 27 de abril de 2021. 
12. MINISTÉRIO DA EDUCAÇÃO. Gabinete do Ministro. Portaria № 343, de 17 de março de 2020. Dispõe sobre a substituição das aulas presenciais por aulas em meios digitais enquanto durar a situação de pandemia do Novo Coronavírus - COVID-19. Diário Oficial da União, Brasília, DF, ed. 53, 18 mar. 2020. Seção 01, p. 39. Disponível em: https://www.in.gov.br/en/web/dou/-/portaria-n-343-de-17-de-marco-de-2020-248564376. Acessado em: 27 de abril de 2021.

13. MINISTÉRIO DA EDUCAÇÃO. Gabinete do Ministro. Portaria № 544, de 16 de junho de 2020. Dispóe sobre a substituição das aulas presenciais por aulas em meios digitais, enquanto durar a situação de pandemia do novo coronavírus - Covid-19. e revoga as Portarias MEC n 343, de 17 de março de 2020, n 345, de 19 de março de 2020, e nำ473, de 12 de maio de 2020. Diário Oficial da União, Brasília, DF, ed., 2020. Seção 1, p.. Disponível em: https://www.in.gov.br/en/web/dou/-/portaria-n-544-de-16-de-junho-de-2020-261924872 Acessado em: 24/09/2021.

14. MINISTÉRIO DA SAÚDE. Secretaria de Atenção à Saúde. Departamento de Atenção Básica. Caderno de atenção domiciliar. Brasília: Ministério da Saúde, 2013. Disponível em: https://bvsms.saude.gov.br/bvs/publicacoes/caderno_atencao_domiciliar_melhor_casa.pdf. Acesso em: 24 de setembro de 2021.

15. NETTO L, SILVA KL. Reflective practice and the development of competencies for health promotion in nurses'training. Revista da Escola de Enfermagem, 2018; 52.

16. RAMÔA HN, et al. Não Somos Robôs: a Afetividade como Processo Pedagógico no Ensino Fundamental II Durante as Aulas Remotas. EaD em Foco, 2020; 10(2): e1306.

17. RAMOS TH, et al. O impacto da pandemia do novo coronavírus na qualidade de vida de estudantes de enfermagem. Revista de Enfermagem do Centro-Oeste Mineiro, 2020; 10: e4042.

18. RIEGEL F, et al. Developing critical thinking in the teaching os Nursing: a challenge in times of Covid-19 pandemic. Escola Anna Nery, 2021; 25(spe): e20200476.

19. SABINO LMM, et al. Uso de tecnologia leve-dura nas práticas de enfermagem: análise de conceito. Aquichan, 2016; 2(16): 230-239.

20. SALES AB, BOSCARIOLI C. Uso de Tecnologias Digitais Sociais no Processo Colaborativo de Ensino e Aprendizagem. Revista Ibérica de Sistemas e Tecnologias de Informação, 2020; 37(6): 82-98.

21. SANTOS GMRF, et al. COVID-19: ensino remoto emergencial e saúde mental de docentes universitários. Revista Brasileira de Saúde Materno Infantil, 2021; 21: 245-251.

22. SILVA MD, et al. Coronavírus: consequências da pandemia no ensino superior. Revista Eletrônica Acervo Saúde, 2021; 13(5): e7120. 